\title{
Anomalies of the Achucarro-Ortiz black hole
}

\author{
Kui Xiad and Wenbiao Livi \\ Department of Physics, Institute of Theoretical Physics, Beijing Normal University, Beijing, 100875, China \\ Hongbao Zhang: \\ Department of Astronomy, Department of Physics, Beijing Normal University, Beijing, 100875, China \\ CCAST (World Laboratory), P.O. Box 8730, Beijing, 100080, China
}

\begin{abstract}
Considering anomalies of quantum field in the $(1+1)$-dimensional Achucarro-Ortiz black hole background, the stress tensor near and out of the horizon is calculated, meanwhile, the relationship between anomalies and Hawking radiation of the black hole is discussed.

Keywords: Achucarro-Ortiz black hole; stress tensor; confromal anomaly; gauge anomaly; gravitation anomaly; Hawking radiation

PACS numbers: 04.62.+v, 04.70.Dy, 11.30.-j
\end{abstract}

\section{INTRODUCTION}

Anomalies are the breaking of classical symmetries by quantum mechanics, and these phenomena are always used to understand a new physics system [1]. Conformal anomaly appears in a theory that the energy momentum has a trace $T_{\alpha}^{\alpha}$ which vanishes classically. The relation between conformal anomaly and Hawking radiation [2] has been studied by many authors [3, 4, 5]. Considering conformal anomaly, many scientists studied the Casimir effect in (1+1)-dimensional curved spacetime background [6, 7].

Gravitational anomaly shows the nonconservation of stress tensor. In (1+1)-dimensional spacetime, the gravitational anomaly is given by [8]

$$
\nabla_{\mu} T_{v}^{\mu}=\frac{1}{96 \pi \sqrt{-g}} e^{\beta \delta} \partial_{\delta} \partial_{\alpha} \Gamma_{\nu \beta}^{\alpha}=\frac{1}{\sqrt{-g}} \partial_{\mu} N_{v}^{\mu}=\mathcal{A}_{v} .
$$

In this field, Robinson and Wilczek discussed the gravitational anomaly in the region that localized on the width of $2 \varepsilon$ straddling the horizon and found that the flux of Hawking radiation cancels the gravitational anomaly [9]. Based on the work of [9], Iso et al calculated the relation between the Hawking radiation of a charged black hole and the gravitational anomaly and gauge anomaly [10]. Many other authors discussed the anomalies near the horizon of different black holes, ie, rotating black hole [11, 12], dynamical black hole [13] and $(2+1)$-dimensional black hole [14].

In this paper, we will calculate the anomalies in $(1+1)$-dimensional Achucarro-Ortiz black hole background. It is organized as following. In Section II, we will introduce the Achucarro-Ortiz black hole in detail. In Section III, the stress tensor in the region $\left(r_{+}+\varepsilon<r<\infty\right)$ is calculated, and then the contribution of trace anomaly to the stress tensor is discussed. In Section IV, we study the gravitational anomaly and gauge anomaly near the horizon, meanwhile, the relation between Hawking radiation and anomalies in this region is discussed. In Section V, we will get conclusions and give some comments.

\section{ACHUCARRO-ORTIZ BLACK HOLE}

The (2+1)-dimensional BTZ black hole was first derived by Banados Teitelboim, and Zanelli [15] under the action

$$
S=\int d^{3} x \sqrt{-g}(R+2 \Lambda)
$$

\footnotetext{
*Electronic address: 87xiaokui@163.com

${ }^{\dagger}$ Electronic address: wbliu@bnu.edu.cn

‡Electronic address: hbzhang@pkuaa.edu.cn
} 
with a negative cosmology constant $\Lambda$. The element of BTZ black hole is

$$
\begin{aligned}
d s^{2}= & -\left(-M+\Lambda r^{2}+\frac{J^{2}}{4 r^{2}}\right) d t^{2}+\left(-M+\Lambda r^{2}+\frac{J^{2}}{4 r^{2}}\right)^{-1} d r^{2} \\
& +r^{2}\left(d \theta-\frac{J}{2 r^{2}} d t\right)^{2} .
\end{aligned}
$$

There are many ways to reduce this line element to $(1+1)$-dimensional one [16, 17]. Under the Kaluza-Klein reduction the metric of BTZ black hole yields the two-dimensional line element as [18]

$$
d s^{2}=-\left(-M+\Lambda r^{2}+\frac{J^{2}}{4 r^{2}}\right) d t^{2}+\left(-M+\Lambda r^{2}+\frac{J^{2}}{4 r^{2}}\right)^{-1} d r^{2},
$$

with a $\mathrm{U}(1)$ gauge field $A_{t}=\frac{J}{2 r^{2}}$ and a dilaton field $\Phi=r$, where $M$ and $J$ are the mass and charge of the (1+1)-dimensional charged black hole. Supposing that the black hole has a positive mass and the charge $J$ is nonzero, there are two horizons of the Achucarro-Ortiz black hole

$$
r_{ \pm}^{2}=\frac{M \pm \sqrt{M^{2}-\Lambda J^{2}}}{2 \Lambda}
$$

where $r_{ \pm}$is the outer and inner horizon respectively. The Hawking temperature $T_{H}$ of the event horizon is [17]

$$
\begin{aligned}
T_{H} & =\frac{\sqrt{2 \Lambda}}{2 \pi} \frac{\sqrt{M^{2}-\Lambda J^{2}}}{\left(M+\sqrt{M^{2}-\Lambda J^{2}}\right)^{\frac{1}{2}}} \\
& =\frac{\Lambda}{2 \pi}\left(\frac{r_{+}^{2}-r_{-}^{2}}{r_{+}}\right),
\end{aligned}
$$

and the Ricci scalar is

$$
R=-\left[2 \Lambda+\frac{3 J^{2}}{2 r^{4}}\right] .
$$

We can rewrite the line element (4) in a conformal form

$$
d s^{2}=\Omega(r)\left(-d t^{2}+d r *^{2}\right),
$$

in which

$$
\Omega=\left(-M+\Lambda r^{2}+\frac{J^{2}}{4 r^{2}}\right), \quad \frac{d r}{d r *}=\Omega .
$$

Then the nonzero Christoffel symbols of the metric $(8)$ are

$$
\Gamma_{t t}^{r *}=\Gamma_{t r *}^{t}=\Gamma_{r * r *}^{r *}=\frac{1}{2} \frac{d}{d r} \Omega .
$$

\section{TRACE ANOMALY}

We consider a massless scalar field in (1+1)-dimensional Achucarro-Ortiz black hole background. The stress tensor is covariantly conserved

$$
\nabla_{v} \Sigma_{\mu}^{v}=0, \quad \Sigma_{\mu}^{\mu}=\frac{1}{24 \pi} R,
$$

in which $\nabla$ is the covariant differentiation operator, $\Sigma_{\mu}^{\mu}$ is the trace anomaly coming from the renormalization process, and $R$ is the curvature scalar.

We assume the background spacetime is steady, then the stress tensor $\Sigma_{\mu}^{v}$ is time independent. Considering the nonzero Christofell symbols (10), the covariantly conserved equation (11) in this background takes the form

$$
\begin{aligned}
\partial_{r *} \Sigma_{t}^{r *}+\Gamma_{t r *}^{t} \Sigma_{t}^{r *}-\Gamma_{t t}^{r *} \Sigma_{t}^{r *} & =0, \\
\partial_{r *} \Sigma_{r *}^{r *}+\Gamma_{t r *}^{t} \Sigma_{r *}^{r *}-\Gamma_{r * t}^{t} \Sigma_{t}^{t} & =0,
\end{aligned}
$$


with $\Sigma_{r *}^{t}=-\Sigma_{t}^{r *}$, and $\Sigma_{t}^{t}=\Sigma_{\alpha}^{\alpha}-\Sigma_{r *}^{r *}$. Using Eqs. (10), (12), (13) one can get that

$$
\begin{aligned}
\frac{d}{d r}\left(\Omega(r *) \Sigma_{t}^{r *}\right) & =0 \\
\frac{d}{d r}\left(\Omega(r *) \Sigma_{r *}^{r *}\right) & =\frac{1}{2}\left\{\frac{d}{d r}(\Omega(r *)\} .\right.
\end{aligned}
$$

Eq.(14) leads to

$$
\Sigma_{t}^{r *}=\alpha \Omega^{-1}(r),
$$

where $\alpha$ is an integration constant. And the solution of Eq. 15 can be written as following

$$
\Sigma_{r *}^{r *}=[H(r)+\beta] \Omega^{-1}, \quad \beta=\Omega(L) \Sigma_{r *}^{r *}(L),
$$

where

$$
H(r)=\frac{1}{2} \int_{L}^{r} \Sigma_{\alpha}^{\alpha} r^{\prime} \frac{d}{d r^{\prime}} \Omega\left(r^{\prime}\right) d r^{\prime},
$$

in which $L$ is an arbitrary scalar length. Given different scalar length $L$, we can get the different contribution of $H(r)$ to the stress tensor. We need to choose $L$ more carefully. It should not include the region that is very near the horizon because there are quantum fluctuation in this area [19]. We consider that the region out of event horizon can be separated into two areas, one is near the horizon $r_{+}<r<\left(L=r_{+}+\varepsilon\right)$ where $\varepsilon$ is a very small length, and the other is $L<r<\infty$.

Using Equations (14) and (15), one can obtain

$$
\Sigma_{\mu}^{v}=\left(\begin{array}{cc}
\Sigma_{\alpha}^{\alpha}(r)-\Omega^{-1}(r) H(r) & 0 \\
0 & \Omega^{-1} H(r)
\end{array}\right)+\Omega^{-1}(r)\left(\begin{array}{cc}
-\beta & -\alpha \\
\alpha & \beta
\end{array}\right) .
$$

The stress tensor $\Sigma_{\mu}^{v}$ can be written in this form

$$
\Sigma_{\mu}^{v}=\Sigma_{\mu}^{(1) v}+\Sigma_{\mu}^{(2) v}+\Sigma_{\mu}^{(3) v}
$$

where

$$
\begin{aligned}
\Sigma_{\mu}^{(1) v} & =\left(\begin{array}{cc}
\Sigma_{\alpha}^{\alpha}(r)-\Omega^{-1}(r) H(r) & 0 \\
0 & \Omega^{-1} H(r)
\end{array}\right), \\
\Sigma_{\mu}^{(2) v} & =K \Omega^{-1}(r)\left(\begin{array}{cc}
-1 & 0 \\
0 & 1
\end{array}\right), \\
\Sigma_{\mu}^{(3) v} & =\alpha \Omega^{-1}(r)\left(\begin{array}{cc}
1 & -1 \\
1 & -1
\end{array}\right),
\end{aligned}
$$

where $K=(\alpha+\beta)$. The next step is to confirm the value of $\alpha$ and $K$. We subject the stress tensor to the condition on which the background spacetime is quasi-flat $r_{q f}$. We can get $\Omega\left(r_{q f}\right) \rightarrow 1, \Sigma_{\alpha}^{\alpha}\left(r_{q f}\right) \rightarrow 0$ in this region. One can compare the energy density of a beam of black body (the Achucarro-Ortiz black hole) radiation in the quasi-flat region with the one of equilibrium gas with Hawking temperature $T_{H}$. The stress tensor of this equilibrium gas is [3]

$$
T_{\mu}^{(e) v}=\frac{\pi}{12}\left(k T_{H}\right)^{2}\left(\begin{array}{cc}
-2 & 0 \\
0 & 2
\end{array}\right) .
$$

Comparing the stress tensor $\Sigma_{\mu}^{(2) v}\left(r_{q f}\right)$ with Eq. (24), we can get

$$
K=\frac{\pi}{6}\left(k T_{H}\right)^{2} .
$$

The outwards flux of thermal radiation in quasi-flat region can be described by the stress tensor

$$
T_{\mu}^{(r) v}=\frac{\pi}{12}\left(k T_{H}\right)^{2}\left(\begin{array}{cc}
-1 & -1 \\
1 & 1
\end{array}\right) .
$$

And the density and the flux are actually equal for a massless field, so that

$$
\alpha=\frac{1}{2}\left[H\left(r_{q f}\right)-\Sigma_{\alpha}^{\alpha}\left(r_{q f}\right)\right] .
$$


Under Eqs. (25) and (27), and considering $K=\alpha+\beta$ and $\beta=\Omega(L) \Sigma_{r^{*}}^{r^{*}}(L)$, one can obtain

$$
\frac{1}{2}\left[H\left(r_{q f}\right)-\Sigma_{\alpha}^{\alpha}\left(r_{q f}\right)\right]=\frac{\pi}{6}\left(k T_{H}\right)^{2}-\Omega(L) \Sigma_{r^{*}}^{r^{*}}(L) .
$$

We find that the total stress tensor $\Sigma_{\mu}^{v}$ is the function of $\Sigma_{\alpha}^{\alpha}, r_{q f}$ and $L . \Sigma_{\alpha}^{\alpha}=\frac{R}{24 \pi}, r_{q f}$ is determined by $g_{t t}=1$, we also can get the value of $L$ from Eq. (28). The stress tensor in this region can be connected to the equilibrium gas of a background heat bath at the temperature of $T_{H}$ and the trace anomaly.

\section{GAUGE ANOMALY AND GRAVITATIONAL ANOMALY}

In this section, we will discuss the stress tensor in the region $r \in\left[r_{+}, r_{+}+\varepsilon\right]$. There are gauge and gravitational anomalies in this region. We consider the current charge and gauge anomaly at first. The current shows an anomaly in the region near the horizon if we omit the ingoing modes in this area. The consistent form of $d=2$ Abelian anomaly of right-handed fields is given by [20]

$$
\nabla_{\mu} J^{\mu}=-\frac{e^{2}}{4 \pi \sqrt{-g}} \epsilon^{\mu v} \partial_{\mu} A_{v}
$$

in which

$$
\epsilon^{\mu v}=\left(\begin{array}{cc}
0 & 1 \\
-1 & 0
\end{array}\right)
$$

But the current $J$ is not covariant, we need to define a new covariant current [21]

$$
\widetilde{J}^{\mu}=J^{\mu}+\frac{e^{2}}{4 \pi \sqrt{-g}} A_{\lambda} \epsilon^{\lambda \mu} .
$$

One can find that the coefficient of $\widetilde{J}$ is twice of $J$. The gauge is anomalistic in the region $r \in\left[r_{+}, r_{+}+\epsilon\right]$, the current satisfies

$$
\partial_{r} J_{(H)}^{r}=\frac{e^{2}}{4 \pi} \partial_{r} A_{t}
$$

and it is conserved $\partial_{r} J_{(o)}^{r}=0$ outside the horizon

$$
\partial_{r} J_{(o)}^{r}=0 .
$$

So we can obtain

$$
\begin{aligned}
J_{(H)}^{r} & =c_{H}+\frac{e^{2}}{4 \pi}\left(A_{t}(r)-A_{t}\left(r_{+}\right)\right), \\
J_{(o)}^{r} & =c_{o},
\end{aligned}
$$

where $c_{o}$ and $c_{H}$ are integration constants.

Under gauge transformations, considering the ingoing mode near the horizon, variation of the effective action is given by $-\delta W=\int d^{2} x \sqrt{-g_{(2)}} \lambda \nabla_{\mu} J_{(2)}^{\mu}$, with a gauge parameter $\lambda$. We separate the current as two parts $J^{\mu}=J_{(o)}^{\mu} \Theta_{+}(r)+J_{(H)}^{\mu} H(r)$, where $J_{(o)}^{\mu}$ is the current of out horizon and $J_{(H)}^{\mu}$ is the one near horizon, and $\Theta_{+}(r)=\Theta\left(r-r_{+}-\epsilon\right), H(r)=1-\Theta_{+}(r)$. The variation near the horizon becomes

$$
0=-\delta W=\int d^{2} x \lambda\left[\delta\left(r-\left(r_{+}+\epsilon\right)\right)\left(J_{o}^{r}-J_{H}^{r}+\frac{e^{2}}{4 \pi} A_{t}\right)+\partial_{r}\left(\frac{e^{2}}{4 \pi} A_{t} H\right)\right] .
$$

The last term is canceled by quantum effects, and it is called the Wess-Zumino term induced by the ingoing modes near the horizon [10]. For the delta-function is non-zero in the region $r \in\left[r_{+}, r_{+}+\epsilon\right]$, the coefficient of this term should vanish

$$
c_{o}=c_{H}-\frac{e^{2}}{4 \pi} A_{t}\left(r_{+}\right),
$$

where $c_{H}$ is the value of the consistent current at the horizon. 
It is necessary to fix the value of current at the horizon to determine the current flow. Since the condition should be gauge covariant, we impose that the coefficient of the covariant current at the horizon should vanish. Considering $\tilde{J}^{r}=J^{r}+\frac{e^{2}}{4 \pi} A_{t}(r) H(r)$, the value of the charge flux should be

$$
c_{o}=-\frac{e^{2}}{2 \pi} A_{t}\left(r_{+}\right)=\frac{e^{2} Q}{4 \pi r_{+}^{2}} .
$$

We now turn to discuss the gravitation anomaly. Remember the $(1+1)$-dimensional gravitational anomaly can be described by Eq.(1). Similar as the calculation of gauge anomaly, we can get the flux of stress tensor near the horizon. The total flux of stress tensor is

$$
a_{o}=\frac{e^{2} Q^{2}}{4 \pi r_{+}^{2}}+N_{t}^{r}\left(r_{+}\right)=\frac{e^{2} Q^{2}}{16 \pi r_{+}^{2}}+\frac{\pi}{12 \beta^{2}} .
$$

We want to find the relation between anomalies near horizon and Hawking radiation. One can consider the flux from black body radiation (Hawking radiation of Achucarro-Ortiz black hole) in the positive $r$ direction with a chemical potential. The Planck distribution in Achucarro-Ortiz black hole is given by [10]

$$
\begin{aligned}
I^{( \pm)}(w) & =\frac{1}{e^{\beta(w \pm c)}-1}, \\
J^{( \pm)}(w) & =\frac{1}{e^{\beta(w \pm c)}+1},
\end{aligned}
$$

for bosons and fermions respectively, where $c=-e A_{t}\left(r_{+}\right)$[2]. $I^{(-)}$and $J^{(-)}$correspond to the distributions for particles with charge $e$. If $(w \pm c)>0$, those distributions are suppressed exponentially, but if $(w \pm c)<0$, we must discuss more deeply. When $(w \pm c)<0$, for bosons superradiance will appear, but for fermions, detailed calculations show that there is no superradiance [22]. We will calculate the flux of fermion for simplicity.

$$
\begin{aligned}
& J^{r}=e \int_{0}^{\infty} \frac{d w}{2 \pi}\left(J^{-}(w)-J^{+}(w)\right)=\frac{e^{2} Q}{4 \pi r_{+}^{2}}, \\
& T_{t}^{r}=\int_{0}^{\infty} \frac{d w}{2 \pi} w\left(J^{-}(w)+J^{+}(w)\right)=\frac{\pi}{12 \beta^{2}}+\frac{e^{2} Q^{2}}{16 r_{+}^{4}} .
\end{aligned}
$$

Compering Eq.(41) with Eq.(38), and Eq.(42) with Eq.(39), we find that the flux derived from blackbody radiation at Hawking temperature cancels the flux of charge and energy.

\section{CONCLUSIONS AND COMMENTS}

We have studie the contribution of different anomalies to stress tensor in different regions in $(1+1)$-dimensional AchucarroOrtiz black hole. In the region of $L<r<\infty$, we find the contribution of trace anomaly to the stress tensor can be connected to the equilibrium gas of a background heat bath at the temperature of $T_{H}$. But in the region near the horizon, gauge anomaly and gravitational anomaly should be considered. To avoid losing the general covariance and gauge invariance at quantum level, the total flow of charge and energy-momentum near the horizon of Achucarro-Ortiz black hole must be equal to the one of blackbody radiation at Hawking temperature. This is different from [14] in which the author integrates out modes in a sandwich surrounding the horizon just like [9]. However, we can get the same result (38) and (39) as [14] in which the BTZ black hole is reduced to $(1+1)$-dimensional black hole.

Anomaly is a key to the deeper understanding of quantum field theory. In [6], the relation between the trace anomaly and Casimir effect is discussed in $(1+1)$ dimensional curved spacetime. There are also many papers in which the relation between black hole entropy and anomaly is discussed [23, 24, 25, 26]. Nugayer [27] had studied the relation between Casimir effect and black hole evaporation two decades ago. Many other people discussed the connection of Casimir effect and black hole thermodynamics [28, 29, 30, 31, 32]. The relation between them will be studied more deeply and be understood more clearly.

\section{Acknowledgments}

Xiao thanks Vagenas, Robinson, Iso, Umetsu and Setare for the useful discussions about their works. We would also like to thank the referee of this paper for pointing out many errors in the original manuscript. 
K. Xiao and W. B. Liu are supported by NBRPC ( Grant No.2003CB716302 ), NSFC ( Grant No.10475013) and H. B. Zhang is supported by NSFC ( Grant No.10533010) .

[1] Jeffrey A. Harvey, arXiv: hep-th/0509097

[2] S. Hawking, Commun. Phys. Math. 43,199(1975).

[3] S. M. Christensen and S. A. Fulling, Phys. Rev. D:15, 2088(1977).

[4] D. Lohiya, J. Phys. A: Math. Phys. 11, 1335(1978).

[5] D. V. Frusaev, Phys. Rev. D:51, R5352(1995).

[6] M. R. Setare, Class. Quantum. Grav. 18, 2097(2001). A. A. Saharian and M. R. Setare, Phys. Lett. B:552 , 119(2003). T.Christodoulakis, G. A. Diamandis, B. C. Georgalas and E.C. Vagenas Phys. Rev. D: 64,124022(2001). M. R. Setare Mod. Phys. Lett. A:20, 441(2005).

[7] X. Q. Gao and J. Y. Zhu, Int.J.Mod.Phys. D:15 (2006) 1473 . K. Xiao and W. B. Liu, Chin. Phys. 15(12), 3044(2006).

[8] R. A. Bertlmann and E. Kohlprath, Ann. Phys. (N.Y.) 288, 137(2001).

[9] S. P. Robinson and F. Wilczek, Phys. Rev. Lett. 95, 011303(2005).

[10] S. Iso, H. Umetsu, and F. Wilczek, Phys. Rev. Lett. 96, 151302(2006).

[11] S. Iso, H. Umetsu, and F. Wilczek, Phys. Rev. D:74, 044017 (2006).

[12] Keiju Murata and Jiro Soda, Phys. Rev. D:74, 044018 (2006).

[13] Elias C. Vagenas and Saurya Das, JHEP 0610, 025(2006).

[14] M. R. Setare, arXiv: hep-th/0608080

[15] M. Banados, C. Teitelboim and J. Zanelli, Phys. Rev. Lett. 69, 1849(1992).

[16] A. Achucarro and M. E. Ortiz, Phys. Rev. D:48, 3600(1993).

[17] A.Kumar and K.Ray, Phys. Lett. B:351, 431(1995).

[18] E. C. Vagenas, Phys. Rev. D:68, 024015(2003), E. C. Vagenas, Int. J. Mod. Phys. D:14 573(2005).

[19] H. Ghafarnejad and H. Salehi, Phys. Rev. D:56, 4633(1997).

[20] R. Bertlmann, Anomalies In Quantum Field Theory(Oxford Science Publications Oxford, 2000).

[21] R. Bardeen and B. Zumino, Nucl. Phys. B:244, 421(1981).

[22] W. Unruh, Phys. Rev. Lett. 31, 1265(1973).

[23] G. Amelino-Camelia and D. Seminara, Class.Quant.Grav.13 881(1996)

[24] S. Carlip, arXiv: ar-qc/0601041

[25] A. Dabholkar, Phys. Rev. Lett. 94, 241301(2005).

[26] P.Kraus and F. Larsen, JHEP, 0509, 034(2005).

[27] R. M. Nugayer, Astrophysics and Space Science, 91, 53(1983).

[28] Sean A. Hartnoll, Phys. Rev. D:70, 0440152004.

[29] F. Belgiorno and S. Liberati, arXiv:gr-qc/9612024

[30] A. Widom, E. Sassaroli, Y. N. Srivastava, J. Swain, arXiv:hep-th/9803013

[31] E. Sassaroli, Y. N. Srivastava, J. Swain and A. Widom, arXiv:hep-th/9805479

[32] Yun Soo Myung, Phys.Lett. B:636, 324(2006). 